\title{
Evaluación clínico-bacteriológico del uso de Cotrimexazol en el tratamiento de la shigellosis en lactantes
}

DRES. VALERIA PRADO ]. *. ANTONIO BAVFI", EDUARDO DONOSO *, SRTA BERTA RAMIRFZ"

En la literatura nacional y extranjera en el último tiempo han aparecido numerosas publicaciones que demuestran que la asociación de Sulfametoxazol con el Trimetoprin, tendría una mayor acción tanto in vivo como in vitro, que las drogas por separado frente a una serie de bacterios Gram + y Gram (-) (1) (2).

Investigaciones de Hitchings (3) han establecido que la acción sinérgica de ambos productos se debería a un efecto inhibitorio simultáneo en la síntesis de ácido tetrahidrofólico por parte de la célula bacteriana, el cual interviene en reacciones biosintéticas esenciales para el metabolismo celular bacteriano.

Por otra parte existen trabajos en la literatura extranjera que demuestran que la adquisición de resistencia por parte de shigella a la Ampicilina y otros antibióticos es relativamente frecuente, probab!emente a través de transferencia del factor de resistencia con otras enterobacteriáceas.

Estos hallazgos se han visto corroborados con nuestra propia experiencia, ya que los estudios de sensibilidad in vitro efectuados con las cepas de shigella aisladas en nuestro medio, muestran que un $24 \%$ de ellas son resistentes a la Ampicilina porque tienen una CIM superior a $6,25 \mathrm{u} / \mathrm{ml}(4)$ (5).

Asimismo en la práctica clínica nos vemos enfrentados a algunos casos en los cuales el tratamiento con Ampicilina fracasa. Por este motivo se decidió en el presente estudio, comparar la acción terapéutica de Ampicilina con la mezcla sulfatrimetoprin en el tratamiento de la shigellosis aguda del lactante. Además para demostrar si existe o no una potenciación in vivo de la sulfa por acción de este derivado pirimidínico se decidió com-

\footnotetext{
* Centro Microblología Area Orienci, Hosp. Calvo Mackenna, Santiago.

* Enfermera Servicio Alta Controlada, Hosp. Calvo Mackenna, Satiago.
}

parar estos resultados con el uso de Sulfadiazina en otros grupo de lactantes con shigellosis aguda.

Por los antecedentes favorables aportados por investigaciones de Mimica y colaboradores (6) efectuadas en población infantil del Area Oriente de Santiago, sobre prevención de diarrea infantil con el uso de lactobacilos acidófilos, se propuso probar además si la dación de una alta cantidad de estos bacterios ( 50 veces mayor que la administrada como prevención) es capaz de modificar favorablemente el curso de la shigellosis aguda.

Otro objetivo específico del presente trabajo fue investigar la incidencia de portadores de shigella en los 4 grupos terapéuticos.

Material y metodo. Se estudiaron 65 lactantes con diarrea aguda en los cuales se había aislado shigella en el coprocultivo. Estos lactantes a medida que fueron identificados ingresaron por azar a uno de los siguientes 4 grupos que involucraba diferentes medidas terapéuticas:

a) $\mathrm{Al}$ primer grupo constituido por 24 lactantes se les administró como tratamiento Ampicilina en dosis de $150 \mathrm{mgr} / \mathrm{kg}$-p/día, por vía I. M. c/6 hrs. durante 7 días.

b) Un segundo grupo, 16 lactantes fue tratado con la mezcla de sulfa y trimetoprin en dosis de $40 \mathrm{mcg}$ de sulfa $/ \mathrm{kg}-/$ día c/12 hrs. por vía oral durante 7 días.

c) Un tercer grupo, 12 lactantes, que recibieron Sulfadiazina en dosis de $100 \mathrm{mgr} / \mathrm{kg}-\mathrm{p} /$ día c/ 6 hrs. por vía oral durante 7 días.

d) Un cuarto grupo, 13 lactantes, al cual se le administró Lactobacilos acidófilos en cantidad de 500.000 millones de bacilos incluidos en $10 \mathrm{ml}$ de leche, en una dosis diaria durante 7 días. 
Para evaluar el resultado de los diferentes tratamientos se consideraron los siguientes parámetros:

a) Duración de la deshidratación, expresado en días con hidratación parenteral.

b) Duración de la diarrea después de iniciado tratamiento.

c) Persistencia de la shigella en las deposiciones, efectuado coprocultivo de control al $7^{\circ}$ día de tratamiento.

Para la pesquisa de portadores se efectuó un estudio prospectivo de estos lactantes, realizando coprocultivos de control a los 30 - 60 - 90 días después de iniciada la enfermedad.

Resultados. Desde el punto de vista estadístico, la comparación de los grupos nos demuestra que no hay diferencias entre ellos según la variable edad, el valor de $\mathrm{X}^{2}$ encontrado no es significativo (Tabla No 1).

\section{T A B L A No 1}

DISTRIBUCION SEGUN EDAD DE 65 LACTANTES CON SHIGELLOSIS

\begin{tabular}{|c|c|c|c|c|c|c|}
\hline 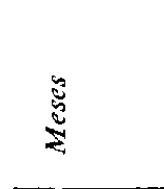 & 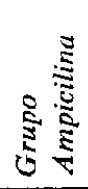 & & 莺 & 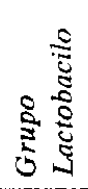 & $N^{o}$ & $\%$ \\
\hline $0-6$ & 16 & 13 & 9 & 12 & 50 & 76,9 \\
\hline $7-24$ & 8 & 3 & 3 & 1 & 15 & 23,1 \\
\hline$T \circ t$ a 1 & 24 & 16 & 12 & 13 & 65 & 100,0 \\
\hline
\end{tabular}

$\mathrm{X}^{2}$ crítico al $5 \%=7,81$.

$\mathrm{X}^{2}$ observado $=3,76 \mathrm{~N}$. S.
T A B L A No 2

DISTRIBUCION SEGUN ESTADO NUTRITIVO DE 65 LACTANTES CON SHIGELLOSIS

\begin{tabular}{lrrrrrr}
\hline &
\end{tabular}

T A B L A No 3

DURACION DE LA DESHIDRATACION EN LACTAVTES CON SHIGELLOSIS SEGUN DIFERENTES MEDIDAS TERAPEUTICAS

\begin{tabular}{lcc}
\hline Tratamiento & $\begin{array}{c}\text { Promedio }(\bar{X}) \\
\text { días con flebo }\end{array}$ & $\begin{array}{c}\text { Lactantes } \\
\text { deshidratados }\left(N^{o}\right)\end{array}$ \\
\hline Ampicilina & 2,7 & 16 \\
Cotrimexazol & 2,9 & 8 \\
Sulfadiazina & 2,0 & 7 \\
Lactobacilo & 2,0 & 8 \\
\hline T o t a 1 & & 39
\end{tabular}

Según el análisis estadístico, no existe evidencia para considerar que los grupos sean diferentes desde el punto de vista del estado nutricional. La diferencia no es estadísticamente significativa al $5 \%$. (Tabla No 2).

19.

T A B L A No 4

ANALISIS ESTADISTICO DE LA DURACION DE LA DESHIDRATACION EXPRESADA EN PROMEDIO DE DIAS DE FLEBO EN LOS 4 GRUPOS CON DISTINTOS TRATAMIENTOS

\begin{tabular}{|c|c|c|c|c|c|c|c|}
\hline & $\bar{X}$ & & $\overleftrightarrow{X}$ & $\begin{array}{c}\text { Grados de } \\
\text { libertad }\end{array}$ & $\begin{array}{l}\text { t critico } \\
\text { al } 5 \%\end{array}$ & tobs. & $\begin{array}{l}\text { Signifi- } \\
\text { cativo }\end{array}$ \\
\hline \multirow{3}{*}{ Ampicilina } & 2,7 & Cotrimexazol & 2,9 & 22 & 2,07 & 0,3 & - \\
\hline & & Sulfadiazina & 2,0 & 21 & 2,08 & 0,53 & - \\
\hline & & Lactobacilos & 2,0 & 22 & 2,07 & 0,56 & - \\
\hline \multirow{2}{*}{ Cotrimexazol } & 2,9 & Sulfadiazina & 2,0 & 13 & 2,16 & 0,55 & - \\
\hline & & Lactobacilos & 2,0 & 14 & 2,15 & 0,60 & - \\
\hline
\end{tabular}


La tabla No 3 muestra el promedio de días que necesitaron hidratación parenteral los lactantes sometidos a las diferentes medidas terapéuticas. El total de niños es inferior al número de estudiados; pues sólo 39 lactantes presentaron deshidratación.

El análisis estadístico muestra que al comparar Ampicilina con los 3 tratamientos no se observan diferencias significativas en la duración de la deshidratación.

Lo mismo resulta al comparar Cotrimexazol con sulfadiazina y lactobacilos. (Tabla No 3 ).

La tabla № 5 muestra el promedio de días de duración de diarrea después de iniciado tratamiento en los 4 grupos de estudio.

Considerando la duración de la diarrea después de iniciado tratamiento, al comparar estadísticamente los resultados observados en el grupo que recibió Ampicilina con relación a los otros 3 grupos terapéuticos no hay diferencias estadísticamente significativas. (Tabla N* 6). Tampoco hay diferencia significativa en la duración de la diarrea en el grupo tratado con Cotrimexazol respecto de los que recibieron Sulfadiazina y Lactobacilos.

En la tabla № 7 vemos el porcentaje de lactantes que mostraba persistencia de shigella en las deposiciones después de 7 días de iniciado el tratamiento correspondiente.

T A B L A NQ 5

DURACION DE LA DIARREA DESPUES DE INICIADO TRATAMIENTO EN LACTANTE CON SHIGELLOSIS CON DIFERENTES MEDIDAS TERAPEUTICAS

\begin{tabular}{lcc}
\hline Tratamiento & $\begin{array}{c}\text { Promedios }(\bar{X}) \\
\text { de diarrea }\end{array}$ & $N^{o}$ de casos \\
\hline Ampicilina & 4,7 & 24 \\
Cotrimexazol & 3,4 & 16 \\
Sulfadiazina & 4,4 & 12 \\
Lactobacilos & 3,3 & 13 \\
\hline
\end{tabular}

T A B L A No 7

PROPORCION DE LACTANTES CON COPROCULTIVO POSITIVO AL $7^{\circ}$ DIA DE TRATAMIENTO. E.N LOS DIFERENTES GRUPOS TERAPEUTICOS

\begin{tabular}{lccc}
\hline Tratamiento & $\begin{array}{c}\text { Coprocultivos } \\
N^{o}\end{array}$ & $\begin{array}{c}\text { positivos } \\
\%\end{array}$ & $\begin{array}{c}\text { Total } \\
\text { estudiados }\end{array}$ \\
\hline Ampicilina & 1 & 4,2 & 24 \\
Cotrimexazol & 2 & 13,3 & 15 \\
Sulfadiazina & 1 & 11,1 & 9 \\
Lactobacilos & 4 & 33,3 & 12 \\
\hline
\end{tabular}

Del análisis estadístico al comparar Ampicili na y los tratamientos en relación a porcentaje de Coprocultivos al $7^{\circ}$ día, vemos que hay diferencias estadísticamente significativas sólo entre Ampicilina $\mathrm{y}^{\prime}$ Lactobacilos con claras ventajas en favor de Ampicilina. (Tabla № 8). Al comparar Cotrimexazol con sulfadiazina no hay diferencias significativas en este sentido. Al efectuar la comparación entre Cotrimexazol y Lactobacilos la diferencia tampoco alcanza significación estadística.

La tabla No 9 expresa los resultados de la pesquisa de portadores de shigella. De los niños controlados sólo en uno apareció shigella en Coprocultivo efectuado a los 90 días.

Comentario. El análisis de los resultados obtenidos demuestra que con cualquiera de los tratamientos usados no se observan diferencias en el curso clínico de la shigellosis, ya que tanto la duración de la diarrea y la duración de la deshidratación es similar en todos ellos, por lo tanto, no hemos demostrado que en este aspecto el Cotrimexazol, quimioterápico en estudio, ni los otros tratamientos ensayados ofrezcan ventajas sobre Ampicilina en el tratamiento de la shigellosis.

Mayor interés revisten los resultados de la evaluación bacteriológica; en este sentido, de acuerdo a lo que nos muestra la tabla 7, vemos que la Ampicilina aparece como la más efectiva para erradicar la shigella, ya que sólo un $4,2 \%$ de los pa-

T A B L A No 6

ANALISIS ESTADISTICO DE LA DURACION DE LA DARREA DESPUES DE INICIADO TRATAMIENTO EN LOS 4 GRUPOS EN ESTUDIO

\begin{tabular}{lclccccc}
\hline & $\begin{array}{c}\vec{X} \text { dias } \\
\text { diarrea }\end{array}$ & & $\begin{array}{c}\vec{X} \text { dias } \\
\text { diarrea }\end{array}$ & $\begin{array}{c}\text { Grados } \\
\text { de libertad }\end{array}$ & $\begin{array}{c}\text { t crítico } \\
\text { al 5\% }\end{array}$ & $\begin{array}{c}\text { tobs. } \\
\text { Signifi- } \\
\text { cativo }\end{array}$ \\
\hline Ampicilina & 4,7 & Cotrimexazol & 3,4 & 37 & 2,04 & 0,69 & - \\
& & Sulfadiazina & 4,4 & 34 & 2,04 & 0,16 & - \\
Cotrimexazol & \multirow{2}{*}{3,4} & Lactobacilos & 3,3 & 35 & 2,04 & 0,84 & - \\
& & Sulfadiazina & 4,4 & 25 & 2,06 & 0,44 & - \\
\hline
\end{tabular}


T A B L A No 8

ANALISIS ESTADISTICO DE LA PROPORCION DE LACTANTES CON PERSISTENCIA DE SHIGELLA EN COPROCULTIVO AL \%? DIA DE TRATAMIENTO

\begin{tabular}{lclcccc}
\hline & $\begin{array}{c}\text { Coproc. } \\
\%\end{array}$ & $\ldots$ & $\begin{array}{c}\text { Coproc. } \\
\%\end{array}$ & $\begin{array}{c}\text { Z crítico } \\
\text { al 5\% }\end{array}$ & $\begin{array}{c}Z \\
\text { obs. }\end{array}$ & $\begin{array}{c}\text { signifi- } \\
\text { cativo }\end{array}$ \\
\hline Ampicilina & 4,2 & Cotrimexazol & 13,3 & 1,65 & 1,03 & - \\
& & Sulfadiazina & 11,1 & 1,65 & 0,7 & - \\
\multirow{3}{*}{ Cotrimexazol } & \multirow{2}{*}{13,3} & Lactobacilos & 33,3 & 1,65 & 2,4 & $\mathrm{X}$ \\
& & Sulfadiazina & 11,1 & 1,65 & 0,16 & - \\
\hline
\end{tabular}

\section{T A B L A No 9}

PESQUISA PROSPECTIVA DE PORTADORES DE SHIGELLA EN LOS 4 GRUPOS TERAPEUTICOS

\begin{tabular}{lcccc}
\hline Tratamiento & $\begin{array}{c}\text { Coprocultivos } \\
\text { efectuados }\end{array}$ & \multicolumn{4}{c}{ Coprocultivos } & Positivos \\
& 33 & 0 & 60 dias & 90 dias \\
\hline Ampicilina & 33 & 0 & 0 & 0 \\
Cotrimexazol & 19 & 0 & 0 & 0 \\
Sulfadiazina & 15 & 0 & 0 & 1 \\
Lactobacilos & 20 & 0 & 0 & \\
\hline
\end{tabular}

cientes muestran persistencia de la shigella en deposiciones al $7^{\circ}$ día de tratamiento, en contraste con un $13,3 \%$ de paciente con shigella en Coprocultivo al $7^{\circ}$ día en el grupo que recibió $\mathrm{Co}$ trimexazol, diferencia que si bien no alcanza significación estadística, cobra importancia desde el punto de vista clínico y epidemiológico, ya que un las diarreas infecciosas el interés está dirigido en forma importante a erradicar el agente infeccioso del intestino, para evitar el problema de infecciones cruzadas.

Lo anteriormente expuesto indica que Ampicilina es tres veces más efectiva en erradicar la shigella del intestino, que el cotrimexazol. Esto difiere totalmente de lo observado in vitro, ya que el estudio de sensibilidad de las cepas de Shigella aisladas en nuestro medio efectuado por B. Lynch (5) muestra que el $100 \%$ de ellas son sensibles al cotrimexazol, y sólo un $76 \%$ lo son a la Ampicilina.

Esta disociación entre el laboratorio y la clinica se debería a nuestro entender, a la dificultad de alcanzar niveles sanguíneos útiles y mantenidos con cotrimexazol, debido a que esta sustancia antimicrobiana se administra por vía oral y la absorción no es óptima en un lactante con diarrea.

Ampicilina en cambio, en nuestros lactantes. fue administrada por vía intramuscular lo cual asegura niveles sanguíneos útiles, necesarios para llegar al lugar donde se localiza la shigella, que es a nivel de la lámina propia de la sub-mucosa, lo cual explicaría los mejores resultados observados en la erradicación de cste agente enteropatógeno.

Respecto al efecto antibacteriano mostrado in vivo de los Lactobacilos acidófilos frente a shigella, vemos en la tabla № 8 que en comparación con ampicilina, hay diferencias altamente significativas en la erradicación de shigella con claras ventajas para Ampicilina, lo cual no hace aconsejable uso de lactobacilos en tratamiento de shigellosis.

Anteriormente habíamos demostrado que en infección enteral por ECEP los lactobacilos acidófilos tampoco tenían efectos terapéuticos satisfactorios, siendo éstos muy inferiores a los obtenidos con el antibiótico de elección neomicina (7) .

De los resultados obtenidos vale la pena destacar que todas las comparaciones entre la combinación de sulfa + trimetoprin denominada cotrimexazol y la sulfadiazina, ya sea tanto en los parámetros de medición clínicos como bacteriológi$\cos$ no se observan diferencias estadísticamente significativas entre ambos tratamientos, luego debemos decir que en shigellosis no hemos podido evidenciar una potenciación in vivo de sulfa + trimetoprin en relación a sulfadiazina.

Los resultados obtenidos con Sulfadiazina en este ensayo terapéutico son bastante satisfactorios y sería el tratamiento de segunde elección después de Ampicilina, hecho que llama profundamente la atención ya que desde la década del 50 se dejó de usar sulfadiazina en shigellosis por la alta incidencia de cepas resistentes observadas, lo cual podría explicar este aparente cambio en la sensibilidad.

Respecto a los resultados de la pesquisa de portadores de shigella realizada en nuestros pacientes, estos fueron totalmente negativos, ya que un paciente en el que se aisló shigella en un coprocultivo a los 90 días, siendo negativos los controles practicados a los 30 y 60 días, correspondería más bien a una nueva infección y no a un portador, aunque no se puede negar la posibilidad 
de falla de la técnica de pesquisa, ya que es de todos conocido el bajo rendimiento del coprocultivo.

De lo anteriormente expuesto podemos concluir que, comparados ampicilina - cotrimexazol, sulfadiazina y lactobacilos en el tratamiento de shigellosis aguda del lactante, es la ampicilina la medida terapéutica que ofrece mayores garantías en la erradicación de la shigella.

\section{RESUMEN}

Se estudiaron 65 lactantes con diarrea aguda a quienes se les aisló Shigella en Coprocultivo. Fueron sometidos a 4 esquemas terapéuticos diferentes.

a) Un grupo de 24 lactantes fueron tratados con Ampicilina por 7 dias.

b) Otro grupo de 16 lactantes recibieron Cotrimexazol durante 7 días.

c) Un tercer grupo, 12 lactantes se les administró Sulfadiazina por 7 días.

d) Un cuarto grupo de 13 lactantes recibieron como tratamiento lactobacilos acidófilos durante 7 dias.

Al efectuar el análisis comparativo de los resultados obtenidos con los 4 esquemas terapéuticos no se apreciaron diferencias significativas en la evolución clínica de la enfermedad, siendo similar la duración de la deshidratación $y$ de la diarrea en todos los grupos.

En cuanto a erradicación de la shigella, la Ampicilina demostró claras ventajas sobre los otros tratamientos. De los lactantes tratados con Ampicilina un $4,2 \%$ mostraba persistencia de shigella en deposiciones a los 7 dias de tratamiento, contra un $13,3 \%$ en el grupo que recibió Cotrimexazol y un $33,3 \%$ en el grupo que recibió lactobacilos.

\section{SUMMARY}

65 hospitalized infants and toddlers with acute diarrhea in whom Shigellae were isolated in the stools were assigned randomly to one of the following treatments:

a) intramuscularly given Ampicilin for seven days.

b) Oral Co-Trimexazole for seven days. c) Orcil Sulfadiazine for seven days.

d) Orally administered lactobacilli for seven days.

There was a stastiscally significative difference between Ampicillin and the other groups regarding stool cultures at the end of the treatment only 4.32 of them remained positive in the Sulphadiazine group, $13.3 \%$ in the Co-trimexazole group and $33.3 \%$ in the lactobacilli group. No statistical differences were found when the four groups were compared regarding the duration of the period of diarrhea, and the duration of the period of dehydration.

From the above mentioned results it can be concluded that intramuscular Ampicillin has clear beneficial effects in children with diarrhea due to shigellosis.

\section{AGRADECIMIENTOS}

Nuestros sinceros agradecimientos a la Sra. Ilse López de Vargas del Irepartamento de Bioestadística de la Escuela de $\mathrm{Sa}$ lubridad, Universidad de Chile, quien realizó el análisis estadístico de los datos.

\section{REFERENCIAS}

1.-Dushley S. R. M.- Trimethoprin-Sulfamethoxazole: In vitro microbiological aspects. J. Infect. Dis. 128 (Suppl) S 442, 1973.

2.-Errázuriz O., Gilardino J., Pino M.-Diarrea aguda del lactante. Estudio clínico-bacteriológico comparativo en grupos tratados con Bactrim, Furoxona y sin medicamento anti-infeccioso intestinal. Rev. Chile. Pecl. Vol. 45, pág. 209, 1974.

3.- Hitchings, C.- Mechanism of action of Trimethoprim-Sulfamethoxazole I. J. Infect. Dis. 128 (Suppl.) S 433,1973

4.- Prado Valeria, Mimica I., Donoso E.- Etiología bacteriana de la diarrea aguda. Aportes de la Inmunofluorescencia directa. Rev. Chile. Ped. Vol. 45. Pág. 135, 1974.

5.-Lynch B., Valenzuela M. C.- Estudio de sensibilidad de Enterobacterias a Cotrimexazol. Presentado a las Jornadas Anuales Pediatría. Valdivia, 1974.

6.-Mimica, I., Lynch B.- Prevención de la diarrea infantil por el uso de lactobacilos. Comunicación personal.

7.-Prado, V., Mimica, I.- Estudio comparativo doble ciego entre los efectos terapéuticos de Neomicina, Lactobacilos versus Placebo en lactantes con infecciónı enteral por ECEP. Rev. Chile Ped. Vol. 45, 1974. 